The risk of ER-negative invasive tumors did not differ between the groups during followup. Although deep-vein thrombosis, pulmonary embolism, vasomotor and gynecological symptoms, and endometrial cancer rates were all higher in the treatment group than in the placebo group during the 5-year treatment period, the rates were comparable in the two groups after cessation of treatment.

These results indicate that the risk:benefit ratio associated with tamoxifen treatment could be much better than previously supposed.

Original article Cuzick J et al. (2007) Long-term results of tamoxifen prophylaxis for breast cancer-96-month follow-up of the randomized IBIS-I trial. J Natl Cancer Inst 99: $272-282$

\section{Study demonstrates new mechanism of action for imatinib}

Imatinib is a tyrosine kinase inhibitor widely used in the treatment of chronic myelogenous leukemia. Originally designed to target the chronicmyelogenous-leukemia-specific tyrosine kinase bcr-abl, imatinib has since been shown to inhibit a number of important tyrosine kinases involved in cell growth and survival. Now, Ertmer et al. have demonstrated a further mechanism through which imatinib might induce growth arrest in cancer cells.

The researchers report for the first time that imatinib can induce cellular autophagy in mammalian cells. Imatinib treatment was shown to activate the lysosomal degradation machinery in a number of different mammalian cell lines, including the mouse neuroblastoma cell line $\mathrm{N} 2 \mathrm{a}$, the monkey kidney cell line COS-7, and the human lung carcinoma cell line A549. Using a green fluorescent protein reporter construct of the autophagy marker LC3, the researchers were able to show that the imatinib-induced upregulation of the lysosomal degradation system was connected with the formation of autophagosomes and activation of cellular autophagy. Further experiments with N2a cells revealed that the induction of autophagosome formation by imatinib was dose-dependent. This result was replicated in nine other mammalian cells lines, as well as in freshly prepared peripheral blood mononuclear cells derived from two human individuals. The authors conclude that imatinib has the ability to induce cellular autophagy, and call for further experiments to investigate this mechanism of action in cancer cells and in vivo.

Original article Ertmer A et al. (2007) The anticancer drug imatinib induces cellular autophagy. Leukemia 21: 936-942

\section{Immunological markers can predict risk of transformation of FL to aggressive DLBCL}

Despite the existence of different treatment options, follicular lymphoma ( $\mathrm{FL})$ is considered incurable. In patients with $\mathrm{FL}$, transformation to aggressive diffuse large B-cell lymphoma (DLBCL) is the major cause of disease-related death. It has been suggested that the outcome in patients with $\mathrm{FL}$ is determined by the interaction of tumor cells with immune cells in the surrounding microenvironment. A study by Glas et al. has confirmed this hypothesis and shown that expression of immunological markers can predict risk of transformation in patients with FL.

The study included samples from 31 patients with FL with proven transformation to DLBCL within 3 years following diagnosis, 35 patients with no signs of transformation 7 years after diagnosis, and 24 patients with DLBCL initially diagnosed with FL. Rapidly transforming FL showed higher levels of expression of immuneresponse-related genes than did nontransforming FL. Gene expression levels in rapidly transforming FL were similar to those in nonmalignant follicular hyperplasia. On the basis of these results, immunohistochemical analyses of protein markers related to function, differentiation and activation of $\mathrm{T}, \mathrm{B}$, natural killer and accessory cells were carried out. No associations were found between $\mathrm{CD}^{+} 8^{+}$macrophages or regulatory $\mathrm{T}$ cells and transformation. By contrast, activation of type $1 \mathrm{~T}$ helper cells was correlated with early transformation $(P=0.004)$ and a role for functional regulation of follicular dendritic cells was also suggested. Moreover, rapidly transforming FL was characterized by the predominant presence of $\mathrm{CD} 4^{+} \mathrm{T}$ cells within the neoplastic follicles. This study shows that immunohistochemistry is more reliable than gene-expression profiling for identification of patients with FL likely to transform to DLBCL.

Original article Glas AM et al. (2007) Gene-expression and immunohistochemical study of specific T-cell subsets and accessory cell types in the transformation and prognosis of follicular lymphoma. J Clin Oncol 25: 390-398 